NASA Technical Memorandum 106433

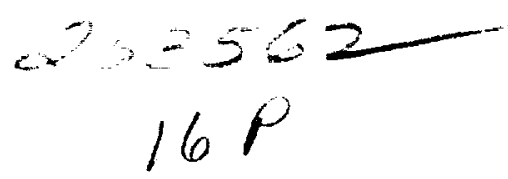

\title{
Multilayer Relaxation and Surface Structure of Ordered Alloys
}

Robert J. Kobistek

Victoreen, Inc.

Solon, Ohio

Guillermo Bozzolo

Analex Corporation

Brook Park, Ohio

John Ferrante

Lewis Research Center

Cleveland, Ohio

and

Herbert Schlosser

Cleveland State University

Cleveland, Ohio

December 1993

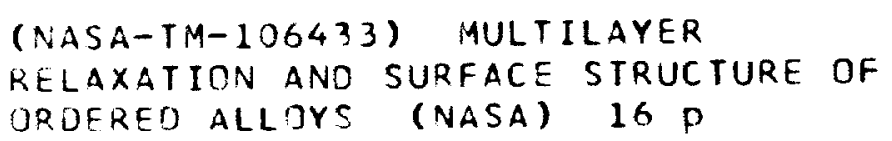

N94-23578

Unclas 
. 


\title{
Multilayer Relaxation and Surface Structure of Ordered Alloys
}

\author{
Robert J. Kobistek \\ Victoreen, Inc. \\ Solon, Ohio 44139 \\ Guillermo Bozzolo \\ Analex Corporation \\ Brook Park, Ohio 44142 \\ John Ferrante \\ Lewis Research Center \\ Cleveland, Ohio 44135 \\ and \\ Herbert Schlosser \\ Cleveland State University \\ Physics Department \\ Cleveland, Ohio 44115
}

\begin{abstract}
Using BFS, a new semiempirical method for alloys, we study the surface structure of fcc ordered binary alloys in the $\mathrm{L}_{2}$ structure $\left(\mathrm{Ni}_{3} \mathrm{Al}\right.$ and $\left.\mathrm{Cu}_{3} \mathrm{Au}\right)$. We show that the surface energy is lowest for the mixed-composition truncation of the low-index faces of such systems. Also, we present results for the interlayer relaxations for planes close to the surface, revealing different relaxations for atoms of different species producing a rippled surface layer.
\end{abstract}




\section{Introduction}

With the recent widespread use of semiempirical methods, as well as the ever-increasing computational capability, there has been an enormous increase in the research in computational material science that, until not long ago, was considered intractable. Surface structure determinations also saw a tremendous development with the advent of new experimental techniques as well as new theoretical approaches for elemental crystals as well as alloys. Recently, much effort has been devoted to the study of surface relaxation in metals and, to a lesser extent, alloys. Several recent experiments have provided insights in the phenomena of surface relaxation and composition, in the case of alloys, and correspondingly a number of theoretical studies have shown good general agreement with experimental results.

However, there is still a great deal of uncertainty in certain areas, due to limitations inherent in experimental techniques and also to the lack of alternative studies to verify previous results. Semiempirical methods are particularly useful tools, providing an efficient and economical way of investigating problems and the consistency of conclusions drawn from experiment.

The wealth of experimental studies of surface relaxation on pure metallic surfaces is not matched for alloys [1]. However, in spite of the small number of experimental studies $[2,3,4]$, there seems to be slow but sure progress in the field, as the available theoretical tools for modelling become more accurate.

The first experiment, in 1984, that provided detailed information on the atomic positions of surface atoms in a truncated ordered alloy is the low-energy electron diffraction (LEED) intensity analysis of Davis and Noonan of a $\mathrm{NiAl}(110)$ surface [2]. They found strong evidence for a rippled surface, where the Al sites of the top laver (in the mixed- 
composition truncation) are displaced above the $\mathrm{Ni}$ sites by approximately $0.22 \AA$. This result was quickly followed by the calculation performed by Chen, Voter and Srolovitz using the embedded atom method (EAM) [5], which confirmed the main features found in the experiment. EAM was later used to investigate similar phenomena in other ordered alloys: Foiles and Daw presented a complete study of $\mathrm{Ni}_{3} \mathrm{Al}\left(\mathrm{L}_{2}\right.$ structure) [6], followed by Foiles work on ordered surface phases of $\mathrm{Au}$ on $\mathrm{Cu}[7]$, and Lundberg's extensive study of surface segregation and relaxation of $\mathrm{Pt}-\mathrm{Ni}$ alloys [8]. At the same time, new experimental LEED results on $\mathrm{Ni}_{3} \mathrm{Al}$ were reported by Sondericker and coworkers [3], finding a similar rippled structure in $\mathrm{Ni}_{3} \mathrm{Al}$ (100) faces. Finally, a low-energy ion scattering spectroscopy (LEISS) experiment by Wang and coworkers provided similar data for the $\mathrm{Cu}_{3} \mathrm{Au}$ system, a classic ordering alloy [4]. Their work followed the LEISS results concerning the surface composition of the top atomic layers [9]. This system was also the subject of a very recent study by Wallace and Ackland using a molecular statics algorithm with Finnis-Sinclair (FS) many-body potentials [10].

The purpose of this work is to provide new results concerning the above mentioned systems, using a new semiempirical method for alloys recently developed by Bozzolo, Ferrante and Smith (BFS) [11], which has shown great promise in previous applications to problems of alloy structure [12]. Due to the simplicity of the method and the ensuing computational efficiency,this application of BFS to the surface structure of ordered alloys can be easily extended to a number of systems for which there are no other theoretical or experimental studies to date [12].

\section{The BFS method}

The BFS method is based on the idea that the energy of formation of an arbitrary 
alloy structure is the superposition of individual contributions $e_{i}$ of non-equivalent atoms in the alloy [12-13]:

$$
e_{i}=e_{i}^{S}+g_{i}\left(e_{i}^{C}-e_{i}^{C_{0}}\right)
$$

$e_{i}$ has two components: a strain energy $e^{S}$ that accounts for the actual geometrical distribution of the atoms surrounding atom $i$, computed as if all its neighbors were of the same atomic species, and a chemical energy $e^{C}$, which takes into account the fact that some of the neighbors of atom $i$ may be of a different chemical species. The coupling function $g_{i}$, ensures the correct asymptotic behavior of the chemical energy contribution. The strain energy of a pure defect crystal is calculated assuming that every neighbor of atom $i$ is of the same species $X . e_{i}^{S}$ is then computed with any technique (first-principles methods, semiempirical techniques,etc.). For $e_{i}^{C}$ we interpret the chemical composition as a defect of an otherwise pure crystal. We represent this defect by 'perturbing' the electronic density in the overlap region between dissimilar atoms and locating them at equilibrium lattice sites of atom $i$. The ideas of equivalent crystal theory [13] are used to develop a procedure for the evaluation of the energy associated with this 'defect'. To free the chemical energy of structural defect energy which should only be included in the strain energy, we reference $e_{i}^{C}$ to a similar contribution where no such perturbation is included $\left(e_{i}^{C_{0}}\right)$. Finally, the coupling function $g_{i}$ is defined as $g_{i}=e^{-a_{i}^{S}}$, where $a_{i}^{S}$ is a solution of $e_{i}^{S}=-E_{C}^{i}\left[1-\left(1+a_{i}^{S}\right) \exp \left(-a_{i}^{S}\right)\right]$, and where $E_{C}^{i}$ is the cohesive energy for atom $i$. We direct the reader to ref. 12 where a detailed description of the calculation of the strain and chemical energy contributions is provided. Except for two parameters determined by fitting to experimental or theoretical alloy properties, the method relies on pure element properties. Moreover, the pairwise character of the interaction between dissimilar species facilitates application to multicomponent 
systems with no further experimental or theoretical input. Within the framework of BFS, the calculation of defect energies requires information on the atomic positions only. The input parameters (see ref. 12 for a complete list) are readily available for a variety of fcc and bcc alloys.

\section{Multilayer relaxation of pure crystals}

Before proceeding to the calculation of multilayer relaxation in alloys, we will discuss some features of theoretical calculations of these quantities. Ref. 1 provides a reasonably large sample of both experimental and theoretical results for changes in interlayer spacing in pure crystals. In all cases, the theoretical techniques used rely either on the use of input data (generally experimentally determined) or on certain approximations for some of the variables of relevance. Necessarily, the results will depend on such choices. Multilayer relaxations involve at best very small changes in position, and correspondingly, comparable changes in surface energy, whose minimization is the criterion used to determine the final interlayer spacings. Thus, the search for a minimum of the surface energy, as accurate as the minimization technique might be, will be strongly influenced by the two factors indicated above: the approximations used and the shallowness of the minimum in the surface energy surface resulting from small changes in the input parameters. As a consequence, to quote just one value for each of the changes in interlayer spacings as is ordinarily done, might not reflect the ambiguities in these calculations. In this paper we adopt a different path: to each theoretical prediction, we will attach an estimate of the possible errors due to any of the reasons mentioned above. Although there is no certain way to determine such errors (after all, the predictions are, within their own framework, exact), we will see that changes on the order of $1 \%$ in the surface energy can generate quite interesting variations 
in the relaxation schemes predicted. In particular, within the framework of ECT, such small changes in the surface energy can be easily obtained by changing any of the input parameters (lattice constant, cohesive energy, bulk modulus) by a similar amount, well below the usual experimental errors in the determination of such quantities.

To illustrate this issue, we will focus our attention on the surface structure of some fcc pure metals ( $\mathrm{Al}, \mathrm{Au}, \mathrm{Cu}$ and $\mathrm{Ni}$ ). As can be seen in Tables 2-11 of ref. 1, previous theoretical and experimental studies show a wide spread in the predictions of the changes in interlayer spacings for the (100) and (110) surfaces. Even results obtained within the same theoretical technique (EAM, ECT) do not agree with each other (due to different fitting procedures of the embedding function in the case of EAM and different input data in both cases). Although there is general qualitative agreement, regarding the contraction or expansion pattern found for successive layers, in some cases the theoretical values show poor agreement with experimental results (see, for example, Al (100)). The ECT results (from refs. 1 and 13) also highlight this inconsistency. The difference between the values obtained in this work and those from previous applications of ECT is easily traceable to slightly different values of some of the input parameters.

\section{Multilayer relaxation of ordered alloys.}

As mentioned above, in order to account for these and other ambiguities in the calculation, we investigated the change in predicted relaxations due to small changes in the rigid surface energy. We thus defined 'error bars' in such way that all the intermediate values so obtained predict variations in surface energies within that tolerance. Needless to say, this range of values does not include all the possible sets $\left(\Delta d_{12}, \Delta d_{23}\right)$ that correspond to surface energies within the allowed values. It is interesting to note, however, that in most 
cases, all the experimental as well as theoretical predictions fall within the range of values obtained in this fashion.

It should be noted that when comparing our theoretical predictions with available experimental results, the error bars quoted in each case are not rigourously comparable. However, we choose to do so with the only purpose of giving a complete description of the results obtainable with ECT (for pure crystals) and BFS (for alloys), once uncertainties in the input parameters are taken into account. To illustrate this point, we first discuss the surface energies and multilayer relaxations of the unreconstructed low-index surfaces of pure $\mathrm{Al}, \mathrm{Ni}, \mathrm{Cu}$ and $\mathrm{Au}$ crystals. In Table 1 we display the ECT predictions for the surface energies and compare the results with typical experimental values for polycrystalline samples $[14,15]$. The agreement is excellent in all cases. We note that experimental values for the surface energies are for polycrystalline surfaces, thus could be strongly dominated by the predominant surface plane.

In table 2 we compare results for the multilayer relaxations of the first two interlayer spacings for those cases for which recent experimental data is available [16-24]. Once again the agreement is excellent, as it was shown in previous ECT studies of surface structure [1]. The inclusion of the theoretical 'error bar', as mentioned above, allows for a better comparison with experiment as it shows that for most cases, small changes in the input parameters of the method suffice to account for the whole range of possible experimental results. The exceptions are $\mathrm{Al}(100)$ and $\mathrm{Al}(111)$, where the outward relaxation of the surface layer has been attributed to an electron promotion effect [25]. Semiempirical methods (ECT, EAM. etc.), unless specifically designed to do so, do not generally allow for such fine electronic structure effects, thus it is not surprising that our results for $\Delta d_{12}$ in these 
cases predict surface layer contractions, even when the 'error bar' is taken into account. For completeness we also include results for the surface relaxation when only that plane is allowed to relax, in order to single out the influence of subsequent interlayer spacing changes on the surface plane. Again, the agreement with available experimental data is very good in all cases.

As mentioned above, there are few theoretical or experimental studies of ordered alloy surfaces [2-10]. In this paper, we focus our attention on two cases $\left(\mathrm{Ni}_{3} \mathrm{Al}\right.$ and $\mathrm{Cu}_{3} \mathrm{Au}$, in the $\mathrm{L} 1_{2}$ structure) which have been the subject of recent studies [3-6]. A complete presentation of the corresponding results for a larger number of ordered structures as well as different binary alloys of both bcc and fcc elements, for which no theoretical or experimental data exists, will be published elsewhere.

a. $C u_{3} A u$ : Table 3 displays the results for the unrelaxed and relaxed surface energies (in ergs $/ \mathrm{cm}^{2}$ ) as obtained with BFS and with FS many-body potentials [10]. Both methods predict, as expected, lower surface energies for the mixed-composition (100) and (110) truncations. This feature has been experimentally proven via a low-energy ion scattering study which detected equal parts of $\mathrm{Cu}$ and $\mathrm{Au}$ in the top layer [9]. ECT and FS results also agree on the relative change in surface energy once the top-most layers are allowed to relax, in spite of the fact that the FS values are $50 \%$ smaller than the BFS ones. As is also to be expected, the surface energies of (100)1:0 and (110)1:0 faces are comparable to the corresponding values for single $\mathrm{Cu}$ crystals. The corresponding relaxations are quoted in table 4. In order to avoid ambiguities in determining the exact atomic positions from the entries in table 4, we present the relaxations as the percentage change in interlayer spacing from the unrelaxed case to the one measured from the relaxed position to the unrelaxed 
location of the plane immediately below. We also include the BFS predictions for the pure $\mathrm{Cu}$ truncations of the (100) and (110) planes. Although it is to be expected that the top layer relaxation will change as deeper layers are allowed to relax, any ensuing changes would be small, not affecting the conclusions drawn from our results.

For the $\mathrm{Cu}_{3} \mathrm{Au}(100)$ 1:1 Cu:Au case, the results in table 4 imply a rippling of $0.148 \pm 0.023$ $\AA$, which amounts to $3.97 \pm 0.62 \%$ of the lattice parameter determined for this alloy $(3.73$ $\AA$ ). This result compares very well with the $3.77 \%$ rippling ( $\mathrm{Al}$ out, $\mathrm{Ni}$ in) obtained using FS potentials [10]. A similar situation is found for the (110) 1:1 Cu:Au surface, where we find the rippling to be $0.135 \pm 0.04 \AA(3.6 \pm 1.1 \%$ of the lattice parameter $)$, whereas FS potentials predict a rather smaller change of $1.9 \%$. For the mixed-composition (111) $3: 1$ $\mathrm{Cu}: \mathrm{Au}$ surface, BFS predicts a rippling of $4.6 \pm 0.4 \%$ thus agreeing with FS results and experimental evidence that the $\mathrm{Au}$ atoms are farther out than the neighboring $\mathrm{Cu}$ atoms in mixed-composition surfaces.

b. $\mathrm{Ni}_{3} \mathrm{Al}$. The surface energies of relaxed $(100),(110)$ and (111) surfaces are shown in Table 5, where we compare our results with the EAM study of Foiles and Daw [6]. As found for the $\mathrm{Cu}_{3} \mathrm{Au}$ case, the mixed-composition truncations always have a lower surface energy. The differences between the EAM and BFS predictions are consistent with previous calculations for pure metals, where the EAM results are typically $50 \%$ lower than the experimental ones. Surface relaxations are indicated in Table 6, using the same format and notation of table 4. From these results we extract the following values for the gap between $\mathrm{Ni}$ and $\mathrm{Al}$ atoms in the mixed-composition (100), (110) and (111) surfaces: $0.12 \pm 0.04 \AA$, $0.07 \pm 0.05 \AA$ and $0.16 \pm 0.03 \AA$, respectively. A similar trend, but with somewhat smaller values for the rippling are obtained from EAM [5]: $0.09 \AA, 0.06 \AA$ and $0.07 \AA$. A different 
EAM calculation [7] predicts a $0.06 \AA$ separation between $\mathrm{Ni}$ and $\mathrm{Al}$ atoms in all three surfaces. Recent LEED data [3] show $\Delta d_{12}(N i)=-2.73 \%$ and $\Delta d_{12}(A l) \sim 0$ (i.e., $\mathrm{Al}$ is displaced outward with respect to $\mathrm{Ni}$ ) and a rippling of $0.02 \pm 0.03 \AA$ for the (100) surface In conclusion, we have applied BFS for the study of multilayer relaxation of specific ordered alloy surfaces for those cases where comparison with experiment and other theoretical approaches is possible. We found our $\mathrm{Cu}_{3} \mathrm{Au}$ results in agreement with experiment and other calculations in that the mixed layer termination had a lower surface energy than the $\mathrm{Cu}$ terminated layer and that surface relaxation studies indicated that there would be rippled surfaces with a preferred outward relaxation for the $\mathrm{Au}$ atoms. For $\mathrm{Ni}_{3} \mathrm{Al}$ similar agreement was obtained for the surface energy as a function of surface composition as well as for the outward relaxation of $\mathrm{Al}$ atoms on the surface. Finally, we have pointed out that considerable caution must be exercised in presenting quantitative results for surface relaxations in that small changes in input parameters can cause substantial quantitative changes due to shallowness of the energy surface. 


\section{References}

${ }^{1}$ A. M. Rodríguez, G. Bozzolo and J. Ferrante, Surf. Sci. 289 (1993) 100, and references therein.

${ }^{2}$ H. L. Davis and J. R. Noonan, Phys. Rev. Lett. 54 (1985) 566.

${ }^{3}$ D. Sondericker, F. Jona and P. M. Marcus, Phys. Rev. B33 (1986) 900.

${ }^{4}$ Z. Q. Whang, Y. S. Li, C. K. C. Lok, J. Quinn, F. Jona and P. M. Marcus, Sol. State Comm. 62 (1987) 181.

${ }^{5}$ S. P. Chen, A. F. Voter and D. J. Srolovitz, Phys. Rev. Lett. 52 (1986) 1308.

${ }^{6}$ S. M. Foiles and M. S. Daw, J. Mater. Res. 2 (1987) 5.

${ }^{7}$ S. M. Foiles, Surf. Sci. 191 (1987) 329.

${ }^{8}$ M. Lundberg, Phys. Rev. B36 (1987) 4692.

${ }^{9}$ T. M. Buck, G. H. Wheatley and L. Marchut, Phys. Rev. Lett. 51 (1983) 43.

${ }^{10}$ W. E. Wallace and G. J. Ackland, Surf. Sci. Lett. 275 (1992) L685.

${ }^{11}$ G. Bozzolo, J. Ferrante and J. R. Smith, Phys. Rev. B45 (1992) 493.

${ }^{12}$ G. Bozzolo and J. Ferrante, Scr. Met. Mat. 26 (1992) 1275; ibid., Phys. Rev. B45 (1992) 12191; ibid., Scr. Met. Mat. 26 (1992) 907; ibid., Phys. Rev. B46 (1992) 8600; G. Bozzolo, B. Good and J. Ferrante, Surf. Sci. 289 (1993) 169.

${ }^{13}$ J. R. Smith and A. Banerjea, Phys. Rev. Lett. 59 (1987) 2451; ibid., Phys. Rev. B37 (1988) 10411; J. R. Smith, T. Perry, A. Banerjea, J. Ferrante and G. Bozzolo, Phys. Rev. B44 (1991) 6444 .

${ }^{14}$ W. R. Tyson, J. Appl. Phys. 47 (1976) 459.

${ }^{15}$ W. R. Tyson and W. A. Miller, Surf. Sci. 62 (1977) 267.

${ }^{16}$ H. L. Davis, J. B. Hannon, K. B. Ray and E. W. Plummer, Phys. Rev. Lett. 68 (1992) 2632 .

${ }^{17}$ J. R. Noonan and H. L. Davis, Phys. Rev. B29 (1984) 4349.

${ }^{18}$ J. R. Noonan and H. L. Davis, J. Vac. Sci. Technol. A8 (1990) 2671.

${ }^{19}$ J. W. M. Frenken, J. F. van der Veen and G. Allan, Phys. Rev. Lett. 51 (1983) 1876. 
${ }^{20}$ S. M. Yalisove, W. R. Graham, E. D. Adams, M. Copel and T. Gustafsson, Surf. Sci. 171 (1986) 400.

${ }^{21}$ J. E. Demuth, P. M. Marcus and D. W. Jepsen, Phys. Rev. 11 (1975) 1460.

${ }^{22}$ R. Mayer, C. Zhang, K. G. Lynn, W. E. Frieze, F. Jona and P. M. Marcus, Phys. Rev. B35 (1987) 3102 .

${ }^{23}$ I. Stensgaard, R. Feidenhans'l and J. E. Sorensen, Surf. Sci. 128 (1983) 281.

${ }^{24}$ S. A. Lindgren, L. Wallden, J. Rundgren and P. Westrin, Phys. Rev. B29 (1984) 576.

${ }^{25}$ P. J. Feibelman, Phys. Rev. B46 (1992) 2532. 


\begin{tabular}{|c|c|c|c|c|}
\hline Technique & $\mathrm{Al}$ & $\mathrm{Cu}$ & $\mathrm{Ni}$ & $\mathrm{Au}$ \\
\hline Exp. [14] & 1200 & 1790 & 2270 & 1560 \\
Exp. [15] & 1140 & 1780 & 2380 & 1500 \\
Exp. [15] & 1180 & 1770 & 2240 & 1540 \\
\hline ECT(100) & 1203 & 2309 & 2982 & 1546 \\
ECT(110) & 1284 & 2373 & 3073 & 1621 \\
ECT(111) & 856 & 1767 & 2274 & 1136 \\
\hline
\end{tabular}

Table 1: Surface energies (in ergs $/ \mathrm{cm}^{2}$ )

\begin{tabular}{|c|c||ccc|c|cc|}
\hline Element & \multicolumn{1}{|c||}{ Face } & \multicolumn{3}{c|}{ Experiment } & ECT & ECT & (two-layers) \\
& & $\Delta d_{12}$ & $\Delta d_{23}$ & Ref. & $\Delta d_{12}$ & $\Delta d_{12}$ & $\Delta d_{23}$ \\
\hline \multirow{3}{*}{$\mathrm{Al}$} & $(100)$ & +1.8 & & {$[16]$} & $-4.68 \pm 1.62$ & $-5.05 \pm 1.58$ & $+3.35 \pm 0.80$ \\
& $(110)$ & $-8.5 \pm 1.0$ & $+5.5 \pm 1.1$ & {$[17]$} & $-8.29 \pm 2.35$ & $-9.53 \pm 3.58$ & $+1.90 \pm 2.24$ \\
& $(111)$ & $+1.7 \pm 0.3$ & $+0.5 \pm 0.7$ & {$[18]$} & $-3.67 \pm 1.21$ & $-3.94 \pm 1.19$ & $+2.75 \pm 0.61$ \\
\hline \multirow{3}{*}{$\mathrm{Ni}$} & $(100)$ & $-3.2 \pm 0.5$ & & {$[19]$} & $-3.53 \pm 1.68$ & $-3.82 \pm 1.68$ & $+2.48 \pm 0.85$ \\
& $(110)$ & $-9.0 \pm 1.0$ & $+3.5 \pm 1.5$ & {$[20]$} & $-6.32 \pm 2.44$ & $-6.55 \pm 3.63$ & $+0.34 \pm 2.24$ \\
& $(111)$ & $-1.2 \pm 1.2$ & & {$[21]$} & $-2.89 \pm 1.29$ & $-3.10 \pm 1.25$ & $+2.12 \pm 0.63$ \\
\hline \multirow{3}{*}{$\mathrm{Cu}$} & $(100)$ & -2.1 & +0.45 & {$[22]$} & $-3.52 \pm 1.74$ & $-3.81 \pm 1.70$ & $+2.47 \pm 0.86$ \\
& $(110)$ & $-7.5 \pm 1.5$ & $+2.5 \pm 1.5$ & {$[23]$} & $-6.31 \pm 2.46$ & $-6.51 \pm 3.83$ & $+0.29 \pm 2.44$ \\
& $(111)$ & $-0.7 \pm 0.5$ & & {$[24]$} & $-2.88 \pm 1.30$ & $-3.10 \pm 1.25$ & $+2.12 \pm 0.63$ \\
\hline
\end{tabular}

Table 2: Surface relaxations of $\mathrm{Al}, \mathrm{Cu}$ and $\mathrm{Ni}$ as percentages of the rigid interplanar spacings.

\begin{tabular}{|c||c|c||c|c|}
\hline \multicolumn{1}{|c||}{ Face } & \multicolumn{2}{c|}{ Finnis-Sinclair } & \multicolumn{2}{c|}{ BFS } \\
& Unrelaxed & Relaxed & Unrelaxed & Relaxed \\
\hline$(100) 1: 1$ & 896 & 865 & 2119 & 1810 \\
$(100) 1: 0$ & 1192 & 1171 & 2478 & 2247 \\
$(110) 1: 1$ & 1051 & 1024 & 2397 & 2337 \\
$(110) 1: 0$ & 1240 & 1173 & 2873 & 2699 \\
$(111) 3: 1$ & 882 & 863 & 1626 & 1577 \\
\hline
\end{tabular}

Table 3: Unrelaxed and relaxed surface energies (in ergs $/ \mathrm{cm}^{2}$ ) of $\mathrm{Cu}_{3} \mathrm{Au}$. 


\begin{tabular}{|c|c|c|c|c|c|c|}
\hline Layer & Atom & $(100) 1: 1$ & $(100) 1: 0$ & $(110) 1: 1$ & $(110) 1: 0$ & $(111) 3: 1$ \\
\hline \multirow{2}{*}{1} & $\mathrm{Cu}$ & $-2.12 \pm 0.41$ & $+5.38 \pm 0.47$ & $-2.45 \pm 1.14$ & $-3.70 \pm 1.02$ & $+14.35 \pm 0.26$ \\
& $\mathrm{Au}$ & $+5.82 \pm 0.81$ & - & $+7.80 \pm 1.80$ & - & $+22.29 \pm 0.53$ \\
\cline { 2 - 7 } 2 & $\mathrm{Cu}$ & $+5.61 \pm 0.37$ & $+12.87 \pm 0.50$ & $+10.14 \pm 1.37$ & $+12.14 \pm 1.37$ & $+21.20 \pm 0.24$ \\
& $\mathrm{Au}$ & - & $+3.23 \pm 1.23$ & - & $-0.70 \pm 1.30$ & $+2.23 \pm 1.92$ \\
\hline
\end{tabular}

Table 4: Surface relaxations of $\mathrm{Cu}_{3} \mathrm{Au} \mathrm{LI}_{2}$ surfaces. See text for definition of the percentage change.

\begin{tabular}{|c|c|c|}
\hline Face & EAM $[6]$ & BFS \\
\hline$(100) 1: 1$ & 1620 & 2852 \\
$(100) 1: 0$ & 1885 & 3168 \\
$(110) 1: 1$ & 1730 & 3117 \\
$(110) 1: 0$ & 1920 & 3964 \\
$(111) 3: 1$ & 1645 & 2411 \\
\hline
\end{tabular}

Table 5: Surface energies of $\mathrm{Ni}_{3} \mathrm{Al}$ (in $\mathrm{ergs} / \mathrm{cm}^{2}$ ).

\begin{tabular}{|c|c|c|c|c|c|c|}
\hline Layer & Atom & $(100) 1: 1$ & $(100) 1: 0$ & $(110) 1: 1$ & $(110) 1: 0$ & $(111) 3: 1$ \\
\hline \multirow{2}{*}{1} & $\mathrm{Ni}$ & $-1.33 \pm 0.70$ & $+3.41 \pm 0.72$ & $-4.63 \pm 1.65$ & $-5.03 \pm 1.31$ & $+8.56 \pm 0.55$ \\
& $\mathrm{Al}$ & $+5.25 \pm 1.60$ & - & $+0.80 \pm 2.85$ & - & $+16.28 \pm 1.40$ \\
\cline { 2 - 7 } 2 & $\mathrm{Ni}$ & $+4.59 \pm 0.63$ & $+8.82 \pm 0.81$ & $+3.55 \pm 2.19$ & $+5.19 \pm 2.40$ & $+13.69 \pm 0.53$ \\
& $\mathrm{Al}$ & - & $+3.39 \pm 1.46$ & - & $+0.92 \pm 2.74$ & $+4.04 \pm 1.53$ \\
\hline
\end{tabular}

Table 6: Surface relaxations of $\mathrm{Ni}_{3} \mathrm{Al} \mathrm{L}_{2}$ surfaces. See text for definition of the percentage change. 

Public reponting burden for this collection of information is estimated to average 1 hour per response, including the time for reviewing instructions, searching existing data sources. gathering and maintaining the data needed, and completing and reviewing the collection of information. Send comments regarding this burden estimate or any other aspect of this collection of information, including suggestions tor reducing lhis burden, to Washinglon Headquants Sevices. Directorate tor Information Oparations and Reports, 1215 Jeflerson Davis Highway, Suite 1204, Arlington. VA 22202-4302, and to the Office of Management and Budget. Papenwork Reduction Project (0704-0188), Washington, DC 20503.

\begin{tabular}{|l|c|c|}
\hline 1. AGENCY USE ONLY (Leave blank) & $\begin{array}{c}\text { 2. REPORT DATE } \\
\text { December } 1993\end{array}$ & $\begin{array}{r}\text { 3. REPORT TYPE AND DATES COVERED } \\
\text { Technical Memorandum }\end{array}$ \\
\hline
\end{tabular}

\section{TITLE AND SUBTITLE}

Multilayer Relaxation and Surface Structure of Ordered Alloys

\section{FUNDING NUMBERS}

WU-505-90-53

\section{AUTHOR(S)}

Robert J. Kobistek, Guillermo Bozzolo, John Ferrante, and Herbert Schlosser

\section{PERFORMING ORGANIZATION NAME(S) AND ADDRESS(ES)}

National Aeronautics and Space Administration

Lewis Research Center

Cleveland, Ohio 44135-3191

9. SPONSORING/MONITORING AGENCY NAME(S) AND ADDRESS(ES)

National Aeronautics and Space Administration

Washington, D.C. 20546-0001
8. PERFORMING ORGANIZATION REPORT NUMBER

\section{E-8275}

10. SPONSORINGMONITORING AGENCY REPORT NUMBER

NASA TM-106433

11. SUPPLEMENTARY NOTES

Robert J. Kobistek, Victoreen, Inc. 6000 Cochran Road, Solon, Ohio 44139-3395; Guillermo Bozzolo, Analex Corporation, 3001

Aerospace Parkway, Brook Park, Ohio 44142, (work funded by NASA Contract 3-25776); John Ferrante, NASA Lewis Research

Center; and Herbert Schlosser, Cleveland State University, Physics Department, Cleveland, Ohio 44115. Responsible person, John Ferrante, (216) 433-6069.

12a. DISTAIBUTIONAVAILABILITY STATEMENT

12b. DISTRIBUTION CODE

Unclassified - Unlimited

Subject Category 26

13. ABSTRACT (Maximum 200 words)

Using BFS, a new semiempirical method for alloys, we study the surface structure of fcc ordered binary alloys in the $\mathrm{Ll}_{2}$ structure $\left(\mathrm{Ni}_{3} \mathrm{Al}\right.$ and $\left.\mathrm{Cu}_{3} \mathrm{Au}\right)$. We show that the surface energy is lowest for the mixed-composition truncation of the low-index faces of such systems. Also, we present results for the interlayer relaxations for planes close to the surface, revealing different relaxations for atoms of different species producing a rippled surface layer.

14. SUBJECT TERMS

Alloys; Surface gold; Copper; Nickel; Aluminum

15. NUMBER OF PAGES

17. SECURITY CLASSIFICATION
OF REPORT
Unclassified

18. SECURITY CLASSIFICATION OF THIS PAGE

Unclassified
19. SECURITY CLASSIFCATION OF ABSTRACT Unclassified
20. LIMITATION OF ABSTRACT 